\title{
Evidence in perinatal medicine: enough of trial and error?
}

A philosophy of practice based on evidence is well established in perinatal medicine and several large randomised controlled trials, organised in the UK, have made an important contribution in this regard. However, it is important to realise that, despite the current imperative in relation to evidence based practice, ${ }^{1}$ many fundamental aspects of perinatal care have not been subjected to a randomised trial. In the UK setting up studies to address such issues has always been difficult as there is only limited infrastructure available to support the process. Furthermore, important changes during the past few years, both within the service and in the public's perception of the Health Service and research, mean that previous approaches may no longer be sustainable.

During the past 20 years there has been a major improvement in the survival of preterm infants that is unlikely to be repeated. As a result the primary outcomes of future trials are likely to be based on either very small improvements in survival (requiring large numbers of infants), or improved morbidity rates (requiring extensive follow up arrangements for large numbers of babies). Therefore, having developed a protocol the organisers of each new randomised trial have to compete for collaborating centres and largely re-invent the necessary administrative structure and follow up arrangements-all at a stage when the study is unfunded. The public have not generally understood the nature and role of trials and some have been the subject of adverse publicity, ${ }^{2}{ }^{3}$ so gaining consent has become increasingly difficult. This problem is compounded when consent is required at or around the time of delivery or when the study involves a child with a life threatening condition. Given that most recruitment and consent are carried out by clinical staff as an "extension" of their normal duties, it is not difficult to understand the recruitment difficulties experienced by most large perinatal trials in the UK over the past five years.

In recognition of this issue, other countries have opted to deal with the problem by establishing formal collaborative networks. Such a model has also worked in the UK for other specialties-for example, the UK children's cancer study group. Should the UK now establish a perinatal trials network? Around the world such networks use a variety of organisational arrangements. In the USA, in at least one network, collaborating units receive additional funding that is linked to satisfactory recruitment and compliance with trial protocols. In other areas collaborative networks exist on a purely voluntary basis. In the former example entry to the network is limited to a group of highly active hospitals while in the latter essentially all interested perinatal hospitals are free to join. If the UK were to develop a network, what would be the correct approach? The Perinatal Trials Service (PTS) of the National Perinatal Epidemiology Unit (NPEU) already offers advice and support in relation to trials. The missing elements are:

- an administrative infrastructure responsible for co ordinating protocol development, data management, and follow up
- a mechanism for establishing a national strategy and prioritisation so that competing trials are not taking place at the same time

- an established network of units committed to running randomised trials

- financial support for collaborating units to ensure recruitment and data collection are efficient

Although there would be additional costs inherent in such a move, it is important to realise there is a great deal of waste incurred by the present approach. Large amounts of time go into full protocol development and grant applications that do not succeed; funded trials fail to achieve recruitment targets, leading to failure of the study or requests for grant extensions; follow up arrangements are regularly re-invented and or duplicated. Eliminating these problems would represent a real saving.

There are, of course, many controversial issues that require wider debate. Should any network be exclusivefor example, limited to 20 large centres-or inclusiveopen to all? Would membership mean that collaboration in every trial supported by the network was mandatory? Where would the "headquarters" be based and how would it be funded? Would funding for units joining the network be available through Culyer (money from central NHS funds given to Trusts to underpin research)? From the administrative point of view, how would those in the collaboration be viewed by university authorities in relation to grant income and publications, both of which would be "owned" by the network?

Despite these concerns, for the moment the key issue is that of principle. Many individuals involved in perinatal trials are aware of mounting difficulties that could be improved. A collaborative network might allow us to move forwards rapidly and the time is right to consider this option carefully both in relation to if, and how, we might take this step. Everyone involved in perinatal care has a role in that debate. However, if there is support then it must be the Royal Colleges of Paediatrics and Child Health and Obstetrics and Gynaecology who, together, take the lead on discussions with the NHS Executive on the ramifications. Given the government's commitment to "evidence" and "clinical effectiveness," it seems inconceivable that the executive would not support such a development if the message from the profession(s) is clearly supportive.

\section{DAVID FIELD}

Professor of Neonatal Medicine

Leicester Royal Infirmary

Leicester LE1 5WW

1 Department of health. A first class service: Quality in the new NHS. London: The Stationery Office, 1998.

2 Jones J. Government sets up inquiry into ventilation trial. BMF 1999;318:553.

3 Anonymous. The perils of paediatric research. [Editorial]. Lancet 1999;353:685. 